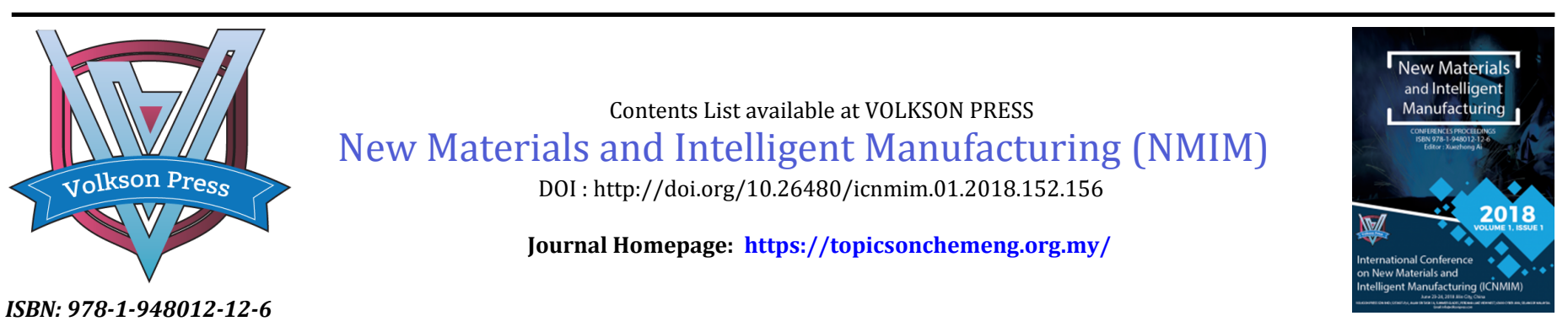

\title{
THE CHITOSAN BASED FLAME RETARDANTS: A MINI REVIEW
}

\author{
Hao Fengling, Geng Weitao, Liu Qun* \\ Jilin Institute of Chemical Technology, Ji Lin, China. \\ *Corresponding Author Email: liuqun198707@163.com
}

This is an open access article distributed under the Creative Commons Attribution License, which permits unrestricted use, distribution, and reproduction in any medium, provided the original work is properly cited

\section{ARTICLE DETAILS}

Article History:

Received 26 June 2018

Accepted 2 July 2018

Available online 1 August 2018

\section{ABSTRACT}

The based Chitosan flame retardant has the characteristics of green sustainable development and meets the future development direction of the flame retardant. Built on the study of the based chitosan flame retardants by scholars at home and abroad, the research progress of the based chitosan flame retardants is systematically introduced in recent years, namely single-component, composite and chemically modified chitosan flame retardants. The singlecomponent chitosan flame retardant can improve the thermal stability of the polymer to a certain extent, but it cannot improve the flame-retardant grade of the material. The main research direction of chitosan composite flame retardant is the application of green compound phosphorus-containing substances, the exploration of different compounding technology, and the in-depth development of layer-by-layer self-assembly technology of fiber products. In the fields of fiber, plastic and other products, the molecular design is based on the requirements of the chitosan flame retardant and the adaptability of the materials, and the characteristics of high efficiency flameretardant, blending compatibility, and finishing wash ability are obtained.

\section{KEYWORDS}

Chitosan, Bio-based, layer-by-layer self-assembly, Chemical modification.

\section{INTRODUCTION}

Greening of flame retardants has been achieved an inevitable trend in the development of flame retardant technology, and the use of natural biobased materials as flame retardants complies with the requirements of the green development strategy. Therefore, the flame retardants prepared with chitosan as raw materials have received extensive attention from researchers.

First of all, chitosan is a nitrogen-containing polysaccharide, biodegradable, biocompatible and environmentally nontoxic. Secondly, the molecular skeleton of chitosan is carbon-rich and contains a certain amount of hydroxyl and amino groups. During the process of thermal decomposition, carbonization prevents combustion and releases noncombustible gas such as $\mathrm{CO}_{2}, \mathrm{NH}_{3}$ and $\mathrm{N}_{2}$, and thus is a flame-retardant effect. Finally, the amino and hydroxyl groups on the chitosan molecule make it get a good reactivity. It can be molecularly designed and introduce multiple flame-retardant elements, so as to amend its physical and chemical properties and improve the flame-retardant efficiency and applicability [1-3]. Chitosan can be used as an antibacterial agent in textiles, plastic, rubber and other products. Especially in the field of fiber products, it was fitted with antibacterial properties and could improve the dyeing, ironing, expansion and stamping resistance [4-6].

\section{SINGLE-COMPONENT CHITOSAN FLAME RETARDANT}

The single-component chitosan flame retardants are characterized by their unique flame-retardant components. Johns modified natural rubber by blending of chitosan. The mass loss after modification was reduced by $43 \%$, and the thermal stability was greatly improved [7]. Don was doped with chitosan in the process of polymerization of methyl methacrylate, the glass conversion temperature was increased from $113^{\circ} \mathrm{C}$ to $127^{\circ} \mathrm{C}$, the amount of carbon residue gradually increased with the increase of the amount of chitosan [8].

The theoretical $\mathrm{C}$ content of chitosan is $45.0 \%$, and the $\mathrm{N}$ content is $8.7 \%$. The modification of the material mainly increases the proportion of carbon in the material and improves the thermal performance. Since the nitrogen content was small, it could only play an auxiliary role in the gas phase flame retardant. Therefore, chitosan can improve the thermal stability of the polymer to a certain extent and made flame retardant performance better, but it cannot improve the flame-retardant grade of the material.

\section{BLENDED CHITOSAN FLAME RETARDANT}

The blended chitosan flame retardant is the compound effect of chitosan and other flame-retardant substances to form multiple phosphorusnitrogen, silicon-phosphorus-nitrogen and other flame-retardant elements. At present, there are mainly poly-phosphate, bio-based materials and inorganic nanoparticles for the blended flame retardants with chitosan.

\subsection{Poly-phosphate}

In the chitosan and poly-phosphate compounding system, chitosan provides carbon source and gas source. Poly-phosphate mainly provide acid source, the blended material can produce a synergistic effect of many flame-retardant elements such as $\mathrm{P}$ and $\mathrm{N}$. Charuchinda used sodium polyphosphate to make cotton fabrics, and it had no flame-retardant effect after washing. On the other hand, the sodium poly-phosphate and chitosan were finished on cotton fabrics. After washing, the washing performance needed to be improved and the LOI was still 19\% [9]. When chitosan and sodium poly-phosphate was used to flame retardant polyester cotton fabric, the system effectively promoted the carbonization of the fabric and self-extinguishing [10]. Carosio applied this technique to polyester cotton fabrics through layer-by-layer self-assembly. The carbon residue at $700^{\circ} \mathrm{C}$ after 20 -layer finishing increasing from $14.7 \%$ to $20.9 \%$ and the total heat release rate decreased from $170 \mathrm{kw} / \mathrm{m}^{2}$ to $128 \mathrm{kw} / \mathrm{m}^{2}$ [11]. Srikulkit deposited poly-phosphoric acid and chitosan on silk fabrics by LbL, the silk was completely decomposed at $600^{\circ} \mathrm{C}$, while 60 layers of silk were covered with coke, and the vein structure was intact [12]. Chen added 5\% ammonium polyphosphate and $2 \%$ chitosan to polylactic acid by melt blending, and the heat release rate was reduced by $51.2 \%$ to UL- $94 \mathrm{~V}-0$ level [13]. Bai added $22.5 \%$ ammonium polyphosphate and $7.5 \%$ chitosan to polypropylene by melt blending. The LOI of composites increased from $18 \%$ to $28.1 \%$, total flue gas release, $\mathrm{CO}$ and $\mathrm{CO}_{2}$ emissions obvious reduction [14].

Chitosan and poly-phosphoric acid (or phosphate) hold a good flame retardant synergistic effect on fibers, plastic and other products, and it 
could be applied to polymer materials with different processing forms through blending, adding and LbL techniques. The film forming properties of chitosan can improve the washability of polyphosphates, and high carbon content and nitrogen source favorably promote the formation of carbonized layers.

\subsection{Bio-based materials}

Bio-based materials have the advantages of wide source, low cost, recyclability and environmental protection, so it used to combine with other matrix of polyelectrolyte properties. At present, the flame retardant of bio-based blended with chitosan mainly include phytic acid, alginate and DNA.

\subsubsection{Phytic acid}

Phytic acid is called inositol six phosphoric acids, which contain six phosphate groups and easy to bind with positively charged molecules. Therefore, the chitosan and phytic acid blended flame retardant often used layer by layer self-assembly technology (LbL) for flame retardant finishing.

Zhang added chitosan and phytic acid to the ethylene-vinyl acetate copolymer, when the added amount was $20 \%$, the flame retardancy of the composite reached UL-94 V-2 [15]. Laufer deposited chitosan and phytate acid onto cotton fabrics by LbL, after 30 layers were deposited, the peak heat release rate and total heat release decreased by $60 \%$ and $76 \%$, and the fabric could be self-extinguished [16]. Xu used chitosan and sodium phytate to form a self-assembled layer on the silk fabric. When 20 layers were assembled, the LOI value of the silk fabric increased from $23.1 \%$ to $31.9 \%$, and after washing 20 time. The LOI value remained at $27.8 \%$ [17].

\subsubsection{Alginate}

Alginate was primarily derived from the brown algae organisms in the ocean. Because alginate has abundant sources, environmental protection and biodegradable, decomposes and absorbs heat, it had certain flameretardant properties.

Chen sorted chitosan and potassium alginate into cotton fabrics through layer by layer self-assembly technology. After 20 layers were deposited, the residual charcoal lines of the flame-retarded cotton fibers were evident, and the expanded char layer appeared on the surface, and there was no afterglow phenomenon [18]. Kumar assembled chitosan, oxidized sodium alginate and phytic acid onto polyamide 66 fabrics by LbL. After deposited 10 layers, the fabric would stop dripping and there would be an expansive char layer on the surface [19].

\subsubsection{Cellulose}

Cellulose is a macromolecular polysaccharide composed of glucose. It is the most widely distributed, most abundant polysaccharide in the natural world and rich in hydroxyl groups and used as a carbon source. Compared with other substances, cellulose is a non-food source and avoids waste. Cellulose can also be modified for different materials by different chemical or physical methods [20].

Mesquita obtained nanocomposites by layer-by-layer assembly (LBL) of highly deacetylated chitosan and cellulose nanowhiskers (CNW) of Eucalyptus. Hydrogen bonding and electrostatic interactions between negatively charged sulfates on the whisker surface and the ammonium groups of chitosan are driving forces for multilayer growth. Scanning electron microscopy showed high density and uniform distribution of CNW adsorbed on each chitosan layer [21]. Pan deposited polyelectrolyte multilayer films of chitosan and phosphorylated cellulose on the surface of cotton fabric by layer-by-layer self-assembly. When the polyelectrolyte multilayer film (PEM) was 20 layers, the peak HRR and THR decreased the most was $70.62 \%$ and $81.25 \%$ lower than that of the pure cotton fabric, and the self-extinguishment occurred. The residual coke at $700^{\circ} \mathrm{C}$ was $35 \%$ [22]. Li prepared polyvinyl alcohol/ nanowhisker cellulose /chitosan (PVA/CNWs/CH) nanocomposites using a water evaporation method. FTIR spectra show that there are hydrogen bonds between PVA and CNW, XRD shows that there are molecular interactions between chitosan and PVA molecules, the elongation at break of PVA/chitosan composites was lower than that of pure PVA. In general, the tensile strength and elongation at break of the PVA/CNWs nanocomposite films are significantly increased compared with the pure PVA films, the enhanced mechanical properties can be attributed to the increase in the interaction between the PVA chains and the CNW via hydrogen bonds. As well as the relatively high strength, stiffness and low density of the CNW, the flexibility of the PVA/CNWs/chitosan nanocomposites compared to the PVA/chitosan films is significantly improved [23].
3.2.4 DNA

DNA is a biological macromolecule consisting of a phosphate group, deoxyribose ring and a nitrogenous base. The molecule contains a large amount of nitrogen and phosphorous, which can serve as a synergistic flame retardant after being decomposed by heat. The unique double helix structure makes it form two single chains when heated, and consumes a lot of heat, so as to achieve a good flame-retardant effect [24,25]. Among chitosan-DNA composite flame retardants, chitosan mainly fixes DNA on the surface of the polymer to form chitosan membrane, so that DNA will not be lost too much in the washing process, thereby enhancing flame retardants and washing resistance.

Annalisa almost completely lost DNA in washing process when DNA was individually flame-retardant cotton fabric. But the flame-retardant effect was nice and showed better water resistance when the DNA and chitosan were finished on the cotton fabric by LbL [26]. Carosio found that selfextinguishing in the horizontal flammability test when the chitosan and DNA flame-retardant were used cotton fabrics, the LOI value increased to $24 \%$, the peak heat release after the deposition of 20 layers decreased by about $41 \%$ and the residual carbon value increased from $2 \%$ to $13 \%$ [27]. At the present moment, the research on blended flame retardation of chitosan and bio-based materials was based on the fact that polyelectrolytes with opposite charges alternate at the liquid/solid interface to form a multilayer film on the surface of the fiber product through electrostatic interaction to achieve durable flame retardant. The layer by layer self-assembly technology (LbL) had the benefits of simple and convenient operation process, environmental protection and adjustable function, which was favorable to the application of the whole bio-based composite flame retardant in fiber products [28].

\subsection{Inorganic nanoparticles}

Nanoparticles have excellent resistance to oxidation and heat such as graphene, montmorillonite, silica, titanium dioxide and zinc oxide. On the basis of chitosan/poly-phosphate and chitosan/bio-based composite systems, the flame-retardant properties of polymer materials have been substantially improved.

\subsubsection{Graphene}

Graphene is an atomic-scale honeycomb lattice structure made up of carbon atoms. Due to its complete lattice structure, graphene has excellent physical barrier effect and higher thermal conductivity [29].

Zhang alternately immersed polyurethane foam in $0.5 \%$ chitosan solution, $0.1 \%$ graphene suspension and $0.3 \%$ alginate solution. After 10 layers were deposited, the peak heat release rate, peak smoke generation rate, total smoke release and carbon monoxide production decreased by $59.9 \%$, $45.6 \%, 30.5 \%, 54.0 \%$. Respectively, the amount of residual carbon increased dramatically [30]. Jin deposited chitosan, grapheme and ammonium polyphosphate onto cotton fabrics through LbL. When 15 layers were assembled, the fabric continued burning time was shortened to $1.5 \mathrm{~s}$ and the damaged length was reduced to $72 \mathrm{~mm}$. The dense carbon layer was formed after the coated fabric burned [31].

\subsubsection{Montmorillonite}

Montmorillonite is a layered silicate crystal consisted of two layers of $\mathrm{Si}-\mathrm{O}$ tetrahedron and one layer of Al-O octahedron. The layer contains sodium, magnesium, calcium, potassium, lithium and other cations. The montmorillonite has good Dispersion and widely used in polymer materials industry, and it could improve impact resistance, fatigue resistance, dimensional stability and gas barrier properties [32].

Laufer applied chitosan and nano-montmorillonite onto polyurethane through layer-by-layer self-assembly technique. The peak heat release rate of the assembled 10 -layer foam was decreased by $52 \%$. In the vertical flame test, this coating completely prevented the flexible polyurethane foam from being ignited [33]. Holder assembled 20 layers of chitosan, ammonium polyphosphate and montmorillonite on polyurethane, the peak exothermic rate decreased by $66 \%$ [34]. Li found that when chitosan, montmorillonite and DNA were assembled onto polyurethane, the maximum heat release rate and average heat release rate decreased by $51 \%$ and $81 \%$ [35].

The tensile strength and thermal stability of polyvinyl alcohol nanometers were improved when chitosan and montmorillonite modified polyvinyl alcohol as electroplating [36]. Li used phytic acid-modified chitosan and melamine phosphate as the synergistic agent to prepare flame-retardant polylactic acid composites through melt blending. Then he found the LOI increased to $30.0 \%$, residual carbon increased to $18.58 \%$, UL-94 reached 
V-0 and melt dripping disappeared [37]. In addition, nano-silica, nanotitanium dioxide and nano-zinc oxide have also been applied to chitosan composite flame retardant, and achieved a certain degree of flame retardant effect [38-41]. In the chitosan/inorganic nanoparticle flame retardant, the inorganic nanoparticle mainly serves as a heat-insulating material protecting matrix, so that the chitosan is not dehydrated in the early stage of the combustion process, water vapor is generated and the coke layer is formed, thereby insulating the air and achieving the goal of flame retardants.

At present, the composite of chitosan and inorganic nanoparticles is mainly through layer-by-layer self-assembly technology and the use of phosphorus-containing polyelectrolyte sandwich assembly to achieve flame-retardant applications in fiber products, polyurethane foam and other materials.

\section{CHEMICALLY MODIFIED CHITOSAN FLAME RETARDANTS}

In addition to the synergistic flame retardants of multi molecular compound, the chitosan based flame retardants have also modified the chitosan structure to form a variety of synergistic flame retardant effects. According to the way of modification, it can be divided into two categories: direct modification and degradation modification.

\subsection{Direct modifications}

The direct modification refers to direct activation of chitosan to enhance reactivity. At present, the main reagents used for direct modification is phosphoric acid and phosphorus pentoxide.

Abou-Okeil used chitosan phosphorylation products to prepare cotton fabrics, after finishing, the decomposition temperature decreased from $350^{\circ} \mathrm{C}$ to $325^{\circ} \mathrm{C}$, the residual carbon value increased from $26.45 \%$ to $36.56 \%$, and the washing resistance improved after finishing [42]. Eltahlawy grafted phosphorylated chitosan onto diammonium phosphate, also used in cotton fabrics. The residual carbon value of the finished fabric was $68 \%$ at $500^{\circ} \mathrm{C}$, while the unfinished fabric left only a small amount of $0.45 \%$ [43].

Phosphorus pentoxide have a higher reactivity than phosphoric acid. Hu reacted chitosan activated with phosphorus pentoxide and urea, then applied it to polyvinyl alcohol fabrics. He found it accelerated the dehydration of the polyvinyl alcohol fabric and formed of a char layer at a low temperature, the residual carbon value of the ruined fabric increased from $0.7 \%$ to $14.3 \%$ at $500^{\circ} \mathrm{C}$ [44]. Subsequently, his research group reacted with chitosan activated with phosphorus pentoxide and melamine again and used in polyvinyl alcohol fabrics, the residual carbon value was increased to $18.5 \%$ and PHRR1 and THR were greatly reduced [45].

\subsection{Degradation modifications}

The degradation modification refers to the method of degrading chitosan into tiny molecules and grafting other flame retardant substances to modify. For the moment, the good flame retardant effects had been achieved on linen and polyurethane fabrics.

Wang reacted degraded chitosan with phosphonitrilic chloride trimer and applied it to linen fabrics. When the content of the flame retardant was $12 \%$, the vertical burning performance of the flax fabric reached the B1 level standard and the B2 level could still be achieved after soaping 10 times [46]. Zhang polymerized carboxymethyl chitosan with sodium pstyrenesulfonate and applied it to the preparation of water-borne polyurethane emulsion. When the flame retardant used less, the mechanical strength of the composite film was changed very small. At the same time, the compatibility with the water-borne polyurethane was nice, so the problem of separating the flame retardant from the flame-retardant matrix material was solved [47]

Chitosan can be chemically modified depending on the requirements of the flame retardant and the adaptability of the material. Through molecular design, the flame retardants introduced a variety of flame retardant elements, which could effectively increase the material's applicability and washability. Through the finishing, blending and other ways to achieve flame retardant effect on fiber products and polyurethane plastic.

\subsection{Problems and development direction of based chitosan flame retardants}

\subsubsection{Problems of based chitosan flame retardants}

(1) The flame-retardant effect of chitosan monomer has a certain degree of flame retardant, but its flame-retardant effect cannot contented the all- round demand of various materials;

(2) The composite based chitosan flame retardant, the flame-retardant effect is better, but it will affect some of the properties of the fabric, such as color, breaking strength, feel.

(3) The research on the application of bio-based the chitosan modification in the field of flame retardant is still in its infancy. There are still many problems that have not been solved, such as:

Bio-based materials mostly contain hydroxyl groups. Although they are good carbon sources, they are also poor in thermal stability and cannot meet the processing requirements of most polymer materials.

The flame-retardant effect of most of the chitosan bio-based flame retardants is not ideal, and it needs to be combined with the flame retardant elements such as phosphorus, nitrogen, and silicon. The modified grafting technology needs to be developed;

A few natural flame-retardant materials such as DNA and phytic acid are expensive and difficult to apply in large quantities;

The bio-based chitosan flame retardants are mostly incompatible with polymer materials and must be modified to improve the overall performance of the material.

\subsubsection{Development direction of based chitosan flame retardants}

(1) Add some flame retardant monomers that have synergistic effect with chitosan and some compatibility with fiber macromolecules in the melt spinning process, so that the fiber macromolecular chains contain flame retardant groups, which is fundamentally method to improve the flame retardant of fabrics, so how to simplify the process and find new flameretardant monomers is still the focus of future research;

(2) The development of novel chitosan modified flame retardant monomers can achieve high flame retardant performance at a low grafting rate and reduce the drawbacks of fabrics with hard and poor feel due to high grafting rates;

(3) Multi-functional modification, Simultaneously we can improve the flame retardant, while giving the fiber other features, such as antibacterial, hydrophilic, skin care and other properties.

(4) The flame retardant finishing process of fabric should meet the requirements of simple process, convenient operation, energy saving and environmental protection;

(5) Relying on bio-based raw materials, adopt reasonable and effective chemical modification technology to overcome the shortcomings of chitosan, while improving the flame-retardant efficiency;

(6) New finishing techniques, such as layer self-assembly method, dualcuring method, nanoparticle adsorption and sol-gel method used to flameretard the polymer and enhance the washing fastness of the substrate.

\section{SUMMARY}

In this mini review, due to the characteristics of high carbon content and low nitrogen content, chitosan could improve the thermal stability of the polymer to a certain extent, and the flame-retardant performance became better. But it could not improve the flame-retardant grade of the material, which was limited as the use of the monocomponent flame retardant.

The blended chitosan flame retardant could be applied to the flameretardant preparation of fibers and plastic products through blending, LbL and ordinary post finishing techniques. The application of green phosphorus-containing substances, the exploration of new compounding technologies and the development of LbL for layered fiber products were important research directions for blended chitosan-based flame retardants.

The chemically modified chitosan-based flame retardants could be molecularly designed based on the requirements of flame retardants and the adaptability of materials, so as to obtain highly effective in flame retardants, blending suitability and finishing wash ability, and it have a broad development space in the fields of fiber and plastic products.

\section{REFERENCES}

[1] Thakur, V.K., Thakur, M.K. 2014. Recent advances in graft copolymerization and applications of chitosan: a review. Acs Sustainable Chemistry \& Engineering, 2 (12), 2637-2652.

[2] Thakur, V.K., Voicu, S.I. 2016. Recent advances in cellulose and chitosan-based membranes for water purification: a concise review. Carbohydrate Polymers, 146 (17), 148-165.

[3] Mural, P.K.S., Kumar, B., Madras, G. 2016. Chitosan immobilized porous polyolefin as sustainable and efficient antibacterial membranes. Acs Sustainable Chemistry \& Engineering, 4 (3), 862-870. 
[4] Croisier, F., Jérôme, C. 2013. Chitosan-based biomaterials for tissue engineering. European Polymer Journal, 49 (4), 780-792.

[5] Li, C., Chenxuan, W. 2017. Green Development Assessment of Smart City Based on PP-BP Intelligent Integrated and Future Prospect of Big Data. Acta Electronica Malaysia, 1 (1), 01-04.

[6] Hejazi, S.M., Lotfi, F., Fashandi, H., Alirezazadeh, A. 2017. Serishm: an eco-friendly and biodegradable flame retardant for fabrics. Environment Ecosystem Science, 1 (2), 05-08.

[7] Johns, J., Rao, V. 2009. Thermal stability, morphology, and x-ray diffraction studies of dynamically vulcanized natural rubber/chitosan blends. Journal of Materials Science, 44 (15), 4087-4094.

[8] Don, T.M., Hu S.C., Chiu, W.Y. 2010. Structures and thermal properties of chitosan-modified poly- (methyl methacrylate). Journal of Polymer Science Part A Polymer Chemistry, 39 (10), 1646-1655.

[9] Charuchinda, S., Srikulkit, K., Mowattana, T. 2005. Co-application of sodium polyphosphate and chitosan to improve flame retardancy of cotton fabric. Sci Res Chula Univ, 30 (1), 97-107.

[10] Guin, T., Krecker, M., Milhorn, A., Grunlan, J.C. 2014. Maintaining hand and improving fire resistance of cotton fabric through ultrasonication rinsing of multilayer nanocoating. Cellulose, 21 (4), 30233030 .

[11] Carosio, F., Alongi, J., Malucelli, G. 2012. Layer by layer ammonium polyphosphate-based coatings for flame retardancy of polyester-cotton blends. Carbohydrate Polymers, 88 (4), 1460-1469.

[12] Srikulkit, K., Iamsamai, C., Dubas, S.T. 2017. Development of flame retardant polyphosphoric acid coating based on the polyelectrolyte multilayers technique. Journal of Metals, 16 (2), 41-45.

[13] Chen, C., Gu, X., Jin, X., Sun, J., Zhang, S. 2017. The effect of chitosan on the flammability and thermal stability of polylactic acid/ammonium polyphosphate biocomposites. Carbohydrate Polymers, 157 (13), 1586

[14] Bai, J., Xue, B.X., Yang, Y.R. 2017. Flame retardancy and smoke suppression of intumescent flame-retardant PP With chitosan/ammonium phosphate [J]. Engineering Plastics Application, 45 (7), 119-123.

[15] Zhang, T., Yan, H., Shen, L. 2014. Chitosan/phytic acid polyelectrolyte complex: a green and renewable intumescent flame-retardant system for ethylene-vinyl acetate copolymer. Industrial \& Engineering Chemistry Research, 53 (49), 19199-19207.

[16] Laufer, G., Kirkland, C., Morgan, A.B. 2012. Intumescent multilayer nanocoating, made with renewable polyelectrolytes, for flame-retardant cotton. Biomacromolecules, 13 (9), 2843-2848.

[17] Xu, J., Yu, P.M., Chen, Z.L. 2014. Technological Condition and Properties Testing of Flame-Retardant Silk Fabrics Prepared by Electrostatic Layer-by-layer Self-assembly Procedure. Science of Sericulture, 40 (1), 75-80.

[18] Chen, X.X., Fang, F., Du, T.X. 2016. Preparation and Properties of Chitosan-Potassium Alginate Flame Retardant Coating via Layer-by-Layer Self-Assembly Technology. Polymer Materials Science \& Engineering, 32 (7), 121-124

[19] Kumar, K.C., Wang, W., Zhou, S. 2017. A green approach to constructing multilayered nanocoating for flame retardant treatment of polyamide 66 fabric from chitosan and sodium alginate. Carbohydrate Polymers, 166, 131-138.

[20] Liang, Z., Xiang, C., Hong, L., Wang, K. 2014. Effects of $\alpha$-blockers, antimuscarinics, or combination therapy in relieving ureteral stentrelated symptoms: a meta-analysis. Journal of Endourology, 29 (6), 650656.

[21] Mesquita, J.P.D., Donnici, C.L., Pereira, F.V. 2010. Biobased nanocomposites from layer-by-layer assembly of cellulose nanowhiskers with chitosan. Biomacromolecules, 11 (2), 473-80.
[22] Pan, H., Song, L., Ma, L., Pan, Y., Liew, K. M., Hu, Y. 2014. Layer-bylayer assembled thin films based on fully biobased polysaccharides: chitosan and phosphorylated cellulose for flame-retardant cotton fabric. Cellulose, 21 (4), 2995-3006.

[23] Li, H.Z., Chen, S.C., Wang, Y.Z. 2015. Preparation and characterization of nanocomposites of polyvinyl alcohol/cellulose nanowhiskers/chitosan. Composites Science and Technology, 115, 60-65.

[24] Mesquita, J.P., Donnici, C.L., Pereira, F.V. 2010. Biobased nanocomposites from layer-by-layer assembly of cellulose nanowhiskers with chitosan. Biomacromolecules, 11 (2), 473-480.

[25] Jia, Y., Hu, Y., Zheng, D. 2017. Synthesis and evaluation of an efficient, durable, and environmentally friendly flame retardant for cotton. Cellulose, 24 (2), 1159-1170.

[26] Casale, A., Bosco, F., Malucelli, G. 2016. Erratum to: dna-chitosan cross-linking and photografting to cotton fabrics to improve washing fastness of the fire-resistant finishing. Cellulose, 23 (3), 1-22.

[27] Carosio, F., Blasio, A.D., Alongi, J. 2013. Green dna-based flameretardant coatings assembled through layer by layer. Polymer, 54 (19), 5148-5153.

[28] Abou-Okeil, A., El-Shafie, A., Hebeish, A. 2007. Chitosan phosphate induced better thermal characteristics to cotton fabric. Journal of Applied Polymer Science, 103 (3), 2021-2026.

[29] Kim, Y.S., Davis, R., Cain, A.A., Grunlan, J.C. 2011. Development of layer-by-layer assembled carbon nanofiber-filled coatings to reduce polyurethane foam flammability. Polymer, 52 (13), 2847-2855.

[30] Zhang, X., Shen, Q., Zhang, X., Pan, H., Lu, Y. 2016. Graphene oxidefilled multilayer coating to improve flame-retardant and smoke suppression properties of flexible polyurethane foam. Journal of Materials Science, 51 (23), 10361-10374.

[31] Jin, Y., Wang, Y. L., Yang, X.C. 2017. Flame Retardant Properties of Cotton with Chitosan-Graphene-Ammonium Polyphosphate coating. Journal of Ceramics, 38 (3), 386-389.

[32] Ray, S.S., Okamoto, M. 2003. Polymer/layered silicate nanocomposites: a review from preparation to processing. Progress in Polymer Science, 28 (11), 1539-1641.

[33] Laufer, G., Kirkland, C., Cain, A. A., Grunlan, J.C. 2012. Clay-chitosan nanobrick walls: completely renewable gas barrier and flame-retardant nanocoatings. Acs Applied Materials \& Interfaces, 4 (3), 16431649.

[34] Holder, K.M., Huff, M.E., Cosio, M.N., Grunlan, J.C. 2015. Intumescing multilayer thin film deposited on clay-based nanobrick wall to produce self-extinguishing flame-retardant polyurethane. Journal of Materials Science, 50 (6), 2451-2458.

[35] Li, Y.C., Yang, Y.H., Kim, Y.S., Davis, R.D. 2014. Dna-based nanocomposite biocoatings for fire-retarding polyurethane foam. Green Materials, 2 (3), 144-152.

[36] Park, J.H., Lee, H.W., Dong, K.C., Oh, W., Yun, J.D., Deng, Y. 2009 Electrospinning and characterization of poly (vinyl alcohol)/chitosan oligosaccharide/clay nanocomposite nanofibers in aqueous solutions. Colloid \& Polymer Science, 287 (8), 943-950.

[37] Li, H., Liu, Z.P., Yang, Z. 2017. Synergistic effect of organic montmorillonite and chitosan-based intumescent flame retardant on flame retardant properties of polylactic acid. China Forestry Science and Technology, 2 (4), 77-83.

[38] Alongi, J., Carosio, F., Malucelli, G. 2012. Layer by layer complex architectures based on ammonium polyphosphate, chitosan and silica on polyester-cotton blends: flammability and combustion behaviour. Cellulose, 19 (3), 1041-1050.

[39] Carosio, F., Alongi, J., Malucelli, G. 2012. Layer by layer ammonium polyphosphate-based coatings for flame retardancy of polyester-cotton blends. Carbohydrate Polymers, 88 (4), 1460-1469. 
[40] Pan, H., Wang, W., Pan, Y., Zeng, W., Zhan, J., Song, L. 2015. Construction of layer-by-layer assembled chitosan/titanate nanotubes based nanocoating on cotton fabrics: flame retardant performance and combustion behavior. Cellulose, 22 (1), 911-923.

[41] Das, K., Maiti, S., Liu, D. 2014. Morphological, mechanical and thermal study of zno nanoparticle reinforced chitosan based transparent biocomposite films. Journal of the Institution of Engineers, 95 (1), 35-41.

[42] Abou-Okeil, A., El-Shafie, A., Hebeish, A. 2007. Chitosan phosphate induced better thermal characteristics to cotton fabric. Journal of Applied Polymer Science, 103 (3), 2021-2026.

[43] Eltahlawy, K. 2008. Chitosan phosphate: a new way for production of eco-friendly flame-retardant cotton textiles. Journal of the Textile Institute, 99 (3), 185-191.

[44] Hu, S., Song, L., Pan, H. 2013. Effect of a novel chitosan-based flame retardant on thermal and flammability properties of polyvinyl alcohol. Journal of Thermal Analysis \& Calorimetry, 112 (2), 859-864.

[45] Lei, S., Yuan, H. 2013. Preparation and characterization of chitosanbased flame retardant and its thermal and combustible behavior on polyvinyl alcohol. Journal of Macromolecular Science: Part D - Reviews in Polymer Processing, 52 (4), 393-399.

[46] Wang, F., Deng, Q.G., Chen, Z.H. 2015. Preparation of degraded chitosan flame retardant containing phosphorus and its application in the treatment of linen fabric. Fine Chemicals, 32 (4), 461-465.

[47] Zhang, L.G. 2016. Research and Application of New Flame Retarded Waterborne Polyurethane Composites, (Thesis dissertation, Southeast University). 